\title{
Ruscogenin attenuated tight junction injury and tumor migration in colorectal liver metastasis mice via regulating TRAP1
}

\author{
Yanni $\mathbf{L v}^{1,2}$, Xuehan $\mathrm{Wu}^{1}$, Jin Chen ${ }^{3}$, Feng Shao ${ }^{1,4}$ \\ ${ }^{1}$ Key Laboratory of Modern Preparation of TCM. Ministry of Education, Jiangxi University of Traditional Chinese Medicine, Nanchang, China; \\ ${ }^{2}$ Department of Pharmacy, The First Affiliated Hospital of Nanchang University, Nanchang, China; ${ }^{3}$ Department of Neurology, The First Affiliated \\ Hospital of Nanchang University, Nanchang, China; ${ }^{4}$ Key Laboratory of Innovation Drug and Efficient Energy-saving Pharmaceutical Equipment, \\ Jiangxi University of Traditional Chinese Medicine, Nanchang, China \\ Contributions: Conception and design: Y Lv, F Shao; (II) Administrative support: All authors; (III) Provision of study materials or patients: All authors; \\ (IV) Collection and assembly of data: X Wu, J Chen, F Shao; (V) Data analysis and interpretation: X Wu, J Chen, F Shao; (VI) Manuscript writing: \\ All authors; (VII) Final approval of manuscript: All authors. \\ Correspondence to: Feng Shao. Associate Professor, Meiling Avenue, No. 1688, Wanli District, Nanchang 330004, China. Email: shaofeng0729@163.com.
}

Background: The effect of ruscogenin on the colorectal cancer is not clear yet. The study was applied to elucidate the mechanism of ruscogenin on colorectal cancer via regulating tumor necrosis factor receptor related protein 1 (TRAP1).

Methods: HCT-116 cells were inoculated under the spleen capsule to establish the colorectal liver metastasis model. The group was divided into control, inoculation model, low dose $(5 \mathrm{mg} / \mathrm{kg})$, mediate dose $(10 \mathrm{mg} / \mathrm{kg})$, and high dose ruscogenin $(20 \mathrm{mg} / \mathrm{kg})$. The body and liver weight of the animals and tumor nodules were recorded. Western blot analysis and immunofluorescence assay were applied to indicate the alternation of tight junction, migration, and proliferation proteins.

Results: Following the inoculated with tumor cells, the mice in the inoculation group suffered from liver volume and weight decrease, as well as the increase of liver tumor volume (TV) and weight (TW). The administration of ruscogenin could obviously decrease body weight and increase liver weight in a dosedependent manner. Meanwhile, 5, 10, $20 \mathrm{mg} / \mathrm{kg}$ ruscogenin could reduce the acreage of tumor nodule on liver, while the high dose $20 \mathrm{mg} / \mathrm{kg}$ ruscogenin could minimize the growth of tumor nodule. The intervention of ruscogenin could relieve the decreased expression of claudin-5, occludin, and ZO- 1 . The administration of ruscogenin could relieve the aggravated tight junction injury by the overexpression of TRAP1, while $20 \mathrm{mg} / \mathrm{kg}$ ruscogenin could not alleviate the tight junction injury already defused by the TRAP1 antibody in the colorectal cancer mice.

Conclusions: Ruscogenin could attenuate the tight junction injury via suppressing TRAP1 in the colorectal cancer mice.

Keywords: Ruscogenin; tight junction; migration; colorectal liver metastasis

Submitted Sep 30, 2020. Accepted for publication Jan 08, 2021.

doi: $10.21037 /$ tcr-20-2968

View this article at: http://dx.doi.org/10.21037/tcr-20-2968

\footnotetext{
^ ORCID: 0000-0002-7976-2643.
} 


\section{Introduction}

Colon cancer is ranked the third of cancer-related death in the world. Symptom of colon cancer at initial stage is not apparent, the pathological process developed quickly since then. The most common options for colon cancer were surgery, chemotherapy, and radiation therapy. Chemotherapy and radiation therapy had common side effects including hair loss, nausea, fatigue, vomiting, etc., while surgery was not effective in treating all tumor sites (1). In the treatment of colon cancer, traditional Chinese medicine Ophiopogon japonicus could play two roles according to its pharmacological action"nourishing yin and tonifying xu" to inhibit tumor metastasis (2). As the main composition of Shengmai injection combined with the injection of oxaliplatin, the liver metastasis model was established by injecting colon cancer cell suspension into the spleen of mice could suppress liver metastasis, significantly reduced the serum related indexes (3). In addition, Shengmai injection has the effect of inhibiting tumor growth and metastasis (4). In the early stage of author's research group, the active components with ruscogenin as the mother nucleus obtained from Ophiopogon japonicus was taken as the research object. It was found that DT-13 and D39, which took ruscogenin as the mother nucleus, could improve TNF- $\alpha$ induced vascular endothelial cells tight junction damage $(5,6)$ and reduce vascular permeability. In particular, DT-13 could significantly inhibit tumor metastasis and invasion of lung cancer A549 cells in vitro by suppressing the expression of MMP-2 and MMP-9 (6).

Most cases of colon cancer cells had invaded healthy tissues nearby that were involved in the spread process of cancer known as metastasis. Metastasis was the feature of the deterioration in the pathological process of cancer disease progression. Metastasis in the final stages of colon cancer development, had been assigned to be more serious and less treatable condition. Colon cancer cells invaded the lymphatics from the primary site, then continued to grow through blood vessels to other places, forming the same type of tumor as the primary site tumor. The most frequently invaded organ is liver (7), about $50 \%$ of patients would have liver metastasis before or after operation, and other invaded organs including lymph metastasis, lung, brain, bone, and other organs. Previous literature has reported that dysfunction of the adherent junction was tightly related with invasion and metastasis of various tumors (8). However, little information was available concerning the tight junction function on animal model associated with colorectal liver metastasis. Tight junctions are composed of several proteins, such as occludin, claudin, ZO-1, ZO-2, and ZO-3. ZO-1 played an important role in the function of the adherent junction of several cancer diseases. Reduced expression of claudin-7induced metastasis and invasion in colorectal cancer via the promotion of epithelial-mesenchymal transition (9). In this study, we investigated the intervention of ruscogenin on the alteration of tight junction and liver metastasis indexes at liver site on colorectal liver metastasis mice.

There existed large numbers of TRAP1 proteins (tumor necrosis factor receptor associated protein 1) in the mitochondria of tumor cells. TRAP1 surrounded the nucleus, with a molecular weight of about $75 \mathrm{kDa}$. TRAP1 could directly combined with the second or fourth complexes of mitochondrial respiratory chain to protect mitochondrial antagonistic antioxidants, and to ensure the survival of tumor cells. Compared with normal cells, TRAP1 in mitochondria of tumor cells increased significantly (10). TRAP1 has been widely reported in ovarian cancer, prostate cancer, and other cancers (11). TRAP1 had a certain effect on the expression of $\mathrm{E}$-cadherin protein, which was the related protein of the epithelial to mesenchymal transition, playing an important role in tumor growth and evolution. However, its role in the progression and metastasis of colorectal cancer was little reported. We examined the effects of ruscogenin on tight junction injury and tumor growth induced by colorectal liver metastasis. The possible mechanisms of ruscogenin might be involved in the intervention of TRAP1. It is of great significance to investigate the mechanism of the active ingredients obtained from the traditional Chinese medicine on colorectal liver metastasis mice. We present the following article in accordance with the ARRIVE reporting checklist (available at: http://dx.doi.org/10.21037/tcr-20-2968).

\section{Methods}

\section{Experimental materials}

SPF grade BALB/c nude female mice were obtained from Weitong Lihua Biotechnology Co., Ltd., laboratory (Beijing, China, No. 1107271911003460). SPF grade $\mathrm{BALB} / \mathrm{c}$ nude female mice, 5 weeks, 16-18 g, 5 animals per cage were raised in SPF level environment. The temperature and humidity was controlled at $20-25^{\circ} \mathrm{C}$ and $40-60 \%$, respectively. Water and bedding were changed 
every week. Experiments were performed under a project license (EA19A139) granted by The First Affiliated Hospital of Nanchang University of ethics committee, in compliance with Animal (Scientific Procedures) Act 1986 guidelines for the care and use of animals. The HCT-116-LUC cells were cultured in RPMI1640 medium containing $10 \%$ fetal bovine serum, which were passed STR short tandem repeat qualification to avoid cross-contaminated or misidentified cell lines (obtained from Wuhan Service Biobiotechnology Co. LTD, China). Experimental equipment: $\mathrm{CO}_{2}$ constant temperature incubator (Panasonic, Japan, mco-18ac), super clean bench (Sujing Antai, China, sw-cj-1fd), biological inverted microscope (Chongqing hydropower Instrument Corporation, China, xds-18), centrifuge (Dalong, China, dm0412), blood cell counting board (Shanghai refining biochemistry, China, 02270113), syringe (Kanglaide, China, U-40). Main experimental reagents: RPMI1640 (Hclone, America, SH30022.01b), fetal bovine serum (seraPro, Australia, s601s-500), trypsin (Amresco, U.S.A., 0457), phosphate-buffered saline (PBS) (Hclone, America).

\section{Cellular culture}

HCT-116 cells transfect with luciferase reporter genes (HCT-116-LUC) were previously established in Wuhan Servicebio biotechnology co. LTD, China laboratory. Both HCT-116 cells and HCT-116-LUC cells were cultured in RPMI1640 medium containing 10\% fetal bovine serum, $10 \%$ antibiotic $(10,000$ units $/ \mathrm{mL}$ penicillin, $10,000 \mu \mathrm{g} / \mathrm{mL}$ streptomycin). Both cell lines were incubated at $37^{\circ} \mathrm{C}$ with $5 \% \mathrm{CO}_{2}$. After growing to a certain amount, trypsin was used to digest and collect cells, PBS was suspended again, and the density was adjusted to $1 \times 10^{7}$ cells $/ \mathrm{mL}$ for inoculation. $1 \times 10^{7}$ cells $/ \mathrm{ml}$ cells suspended in $0.1 \mathrm{~mL}$ PBS were collected in a glass bottle, and then put on ice until inoculation.

\section{Inoculation}

The model of liver metastasis was established in healthy $\mathrm{BALB} / \mathrm{c}$ nude mice. After anesthesia, lay the mice on their sides and opened the abdominal cavity layer by layer, exposing the dorsal side of the spleen. HCT116 cells or HCT-116-LUC cells were inoculated under the spleen capsule. The cells suspended in $0.1 \mathrm{~mL}$ PBS with $1 \times 10^{7}$ cells $/ \mathrm{mL}$ were injected parallel to the spleen. Then sutured the abdomen layer by layer and disinfected the wound with iodine. After waking up, the mice were observed with efficacy indexes and observation indicators every day. The day of tumor cell inoculation was defined as day 0 . Six weeks after cell injection, the mice were killed by $\mathrm{CO}_{2}$ (12). The tumor inoculated mice were subjected to take photos, and the liver or spleen with removal tumor were also subjected to take photos and weigh under Xenogen IVIS Lumina series II. The removal tumor part was stored in liquid nitrogen, or was fixed and embedded.

\section{Animal group}

Protocol one: animals were divided into five groups, which were control, inoculation group, low dose ruscogenin, mediate dose ruscogenin, and high dose ruscogenin. Inoculation group were treated as the inoculation protocol. Ruscogenin was dissolved in $0.5 \%$ sodium carboxymethyl cellulose at a dose of $5,10,20 \mathrm{mg} / \mathrm{kg}$. Mice were given ruscogenin or equal volume of $0.5 \%$ sodium carboxymethyl cellulose by intragastric administration for $1 \mathrm{~h}$ before inoculation. Additional injection of $5,10,20 \mathrm{mg} / \mathrm{kg}$ ruscogenin was given every $24 \mathrm{~h}$ during the inoculation experimental period (13). The groups in protocol one were subjected to monitoring indicators, western blot, and immunofluorescence assay. Protocol two: animals were divided into six groups, which were control, inoculation group, injection of TRAP1 agonists with the inoculated model, injection of TRAP1 agonists with the intervention of ruscogenin with the inoculated model, injection of TRAP1 antagonists with the inoculated model, and injection of TRAP1 antagonists with the intervention of ruscogenin with the inoculated model. Ruscogenin dissolved in $0.5 \%$ sodium carboxymethyl cellulose was $20 \mathrm{mg} / \mathrm{kg}$ intragastric administration for $1 \mathrm{~h}$ before inoculation. TRAP 1 agonists or TRAP1 antagonists were administrated via tail intravenous in $200 \mu \mathrm{L}$ PBS $0.5 \mathrm{~h}$ before inoculation. $20 \mathrm{mg} / \mathrm{kg}$ TRAP1 agonists or antagonists were given at a fixed time per day during the inoculationexperimental period. Injection of TRAP1 agonists could induce the overepxression of TRAP1, which could be explored the role of TRAP1 in colorectal liver metastasis mice $(14,15)$. Injection of TRAP1 antagonists could induce the downepxression of TRAP1, the groups in protocol two were subjected to monitoring indicators, western blot, and immunofluorescence assay. Also, the intervention of ruscogenin was considered under the function of TRAP1.

\section{Efficacy index}

Relative TGI (tumor inhibition rate, \%): TGI = 1 - T/C (\%). 
$\mathrm{T} / \mathrm{C} \%$ was the tumor increment rate, which referred as the percentage ratio of tumor volume (TV) or tumor weight (TW) between the treatment group and the control group at a certain time point. $\mathrm{T}$ and $\mathrm{C}$ was $\mathrm{TV}$ or $\mathrm{TW}$ at a specific time point in the treatment group and the control group, respectively. The calculation formula was as follows: $\mathrm{T} / \mathrm{C} \%=\mathrm{T}_{\mathrm{TW}} / \mathrm{C}_{\mathrm{TW}} \times 100 \%$ ( $\mathrm{T}_{\mathrm{TW}}$ : average $\mathrm{TV}$ in the treatment group at the endpoint of the experiment; $\mathrm{C}_{\mathrm{TW}}$ : average $\mathrm{TV}$ in the control group at the endpoint of the experiment), or $\mathrm{T} / \mathrm{C} \%=\mathrm{T}_{\mathrm{TW}} / \mathrm{C}_{\mathrm{TW}} \times 100 \%$ ( $\mathrm{T}_{\mathrm{TW}}$ : average $\mathrm{TW}$ in the treatment group at the end of the experiment; $\mathrm{C}_{\mathrm{TW}}$ : average $\mathrm{TW}$ in the control group at the end of the experiment). Tumor growth inhibition rate $<40 \%$ was identified as invalid inoculation. Tumor growth inhibition rate $\geq 40 \%$, which met the requirements of $\mathrm{P}<0.05$ was identified as the effective inoculation tumor model.

\section{Observation indicators}

After administration, the state of the animals: activity, hair color, diet, weight were observed and recorded. Body weight before and after each inoculation was measured twice a week during the efficacy observation. Mental state, activity reduction, rough hair, paralysis, and dyspnea indicators of mice were measured during inoculation.

\section{Hematoxylin and eosin staining}

After the liver was removed, it was fixed in cold $4 \%$ paraformaldehyde at $4{ }^{\circ} \mathrm{C}$ for $24 \mathrm{~h}$. Following dehydration, the specimens were embedded in paraffin, cut into $5 \mathrm{~mm}$ sections and stained with H\&E. The histological morphology of each liver specimen was examined under optical microscopy.

\section{Immunocytochemistry}

Liver tissues were washed with $\mathrm{pH}$ 7.4PBSand immobilized with cold ethanol at $4{ }^{\circ} \mathrm{C}$ for $10 \mathrm{~min}$, and immobilized with $10 \%$ normal goat serum, $3 \%$ bovine serum albumin, and $0.1 \%$ Triton X-100 in PBS for $1 \mathrm{~h}$. Then incubated with the anti-TRAP1 (CST, USA, 13405, 1:1,000 for WB, 1:200 for IF, green), anti-occludin (Invitrogen, USA, 33-1500, 1:1,000 for WB, 1:200 for IF, green), anti-claudin-5 (Invitrogen, USA, 34-1600, 1:1,000 for WB, 1:200 for IF, green), anti-ZO-1 (Invitrogen, USA, 40-2300, 1:1,000 for WB, 1:200 for IF, green), anti-ki67 (CST, USA, 9449, 1:1,000 for WB, 1:200 for IF, green), and anti-E-cadherin (CST, USA, 14472, 1:1,000 for WB, 1:200 for IF, red) primary antibody for $48 \mathrm{~h}$ at $4^{\circ} \mathrm{C}$. After washing, cells separately were incubated overnight with Alexa Fluor ${ }^{\circledR} 488$ conjugated Donkey Anti-Goat IgG $(\mathrm{H}+\mathrm{L})$ antibody (Abcam, UK, ab150129, 1:1,000) at $4{ }^{\circ} \mathrm{C}$. The wash step was repeated, the nuclei of all cells were then washed and incubated with 4',6-diamidino-2-phenylindole (DAPI, CST, USA, 4083, $1: 1,000)$ for $15 \mathrm{~min}$ at room temperature. Briefly, confocal images were converted to 8-bit format, and then noise speckle removal was performed to eliminate the singlepixel background fluorescence. Images were collected by a confocal fluorescence microscope (Leiss LSM710) with a magnification of $\times 200$.

\section{Western blot analysis}

The protein concentration was determined using the BCA assay. Samples containing $40 \mathrm{mg}$ protein were separated by $12.5 \%$ SDS-PAGE electrophoresis. The proteins were transferred to PVDF membranes in tris-glycine transfer buffer subsequently. The membranes were blocked for $2 \mathrm{~h}$ with $5 \%$ nonfat dry milk at room temperature and probed with rabbit anti-TRAP1 (1:1,000, CST, USA), antioccludin (1:1,000, Invitrogen, USA), anti-claudin-5 (1:1,000, Invitrogen, USA), anti-ZO-1 (1:1,000, Invitrogen, USA), anti-ki67 (1:1,000, CST, USA), anti-E-cadherin (1:1,000, CST, USA), anti-GAPDH $\left(1: 1,000\right.$, CST, USA) at $4{ }^{\circ} \mathrm{C}$ overnight, and then incubated with the appropriate HRPconjugated secondary antibody for $50 \mathrm{~min}$. The bands were displayed with enhanced immunofluorescence and images were captured using a bio-Image Analysis System (Bio-Rad, Hercules, USA).

\section{Statistical analysis}

Data was analyzed with GraphPad Prism5. All values were expressed as the mean \pm SD. One-way ANOVA followed by Tukey's post-hoc test was used to compare the differences between groups. The unpaired student's $t$-test was used to compare data between two groups. The significant level was set at $\mathrm{P}<0.05$ and $\mathrm{P}<0.01$.

\section{Results}

\section{Ruscogenin attenuated organ indicators in colorectal liver metastasis mice}

At the early stage of tumor cell inoculation, no significant changes were found in spirit, diet, activity, fur, and excreta 

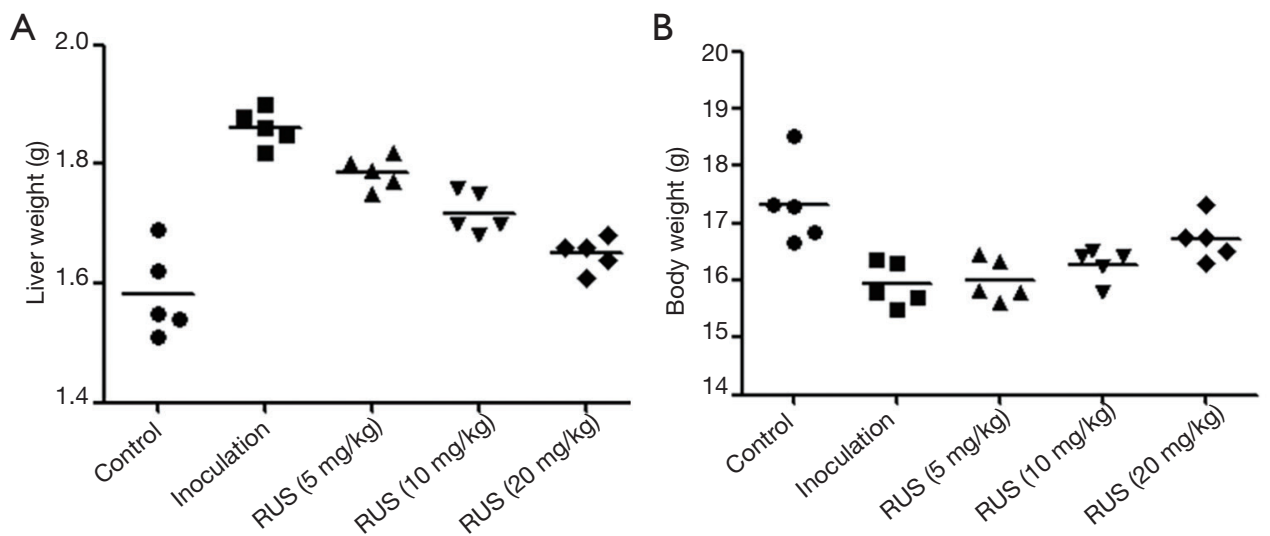

Figure 1 Ruscogenin attenuated body and liver weight in colorectal liver metastasis mice. (A) Ruscogenin attenuated body weight in colorectal liver metastasis mice. (B) Ruscogenin attenuated liver weight in colorectal liver metastasis mice.

Control

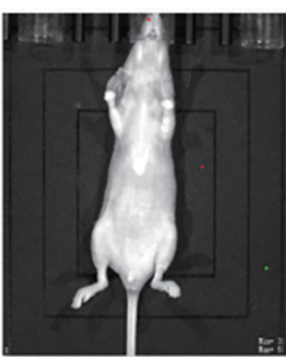

Inoculation

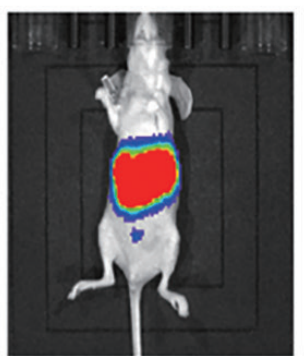

$\operatorname{RUS}(5 \mathrm{mg} / \mathrm{kg})$

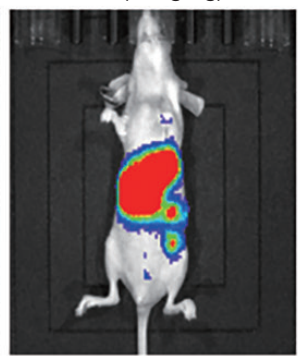

RUS $(10 \mathrm{mg} / \mathrm{kg})$

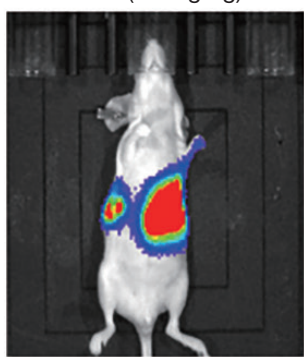

$\operatorname{RUS}(20 \mathrm{mg} / \mathrm{kg})$

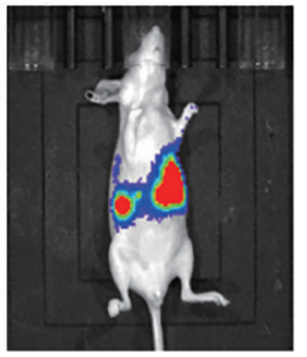

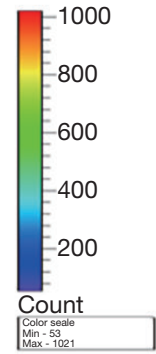

Figure 2 Ruscogenin reduced luciferase bioluminescent signal in colorectal liver metastasis mice.

in all group of mice. At the second week of modeling, the mice in the model group suffered from mental depression, decreased food intake, sluggish action, dry fur, loose stool, and obvious ascites, while the mice in the control group had no obvious change. After 3-4 weeks of inoculation, some of the mice in the control group showed the above situation, but the overall growth state was significantly better than that in the model group. In the model group, metastasis was found in the liver, the rate of liver metastasis was $100 \%$, accompanied by the decrease of body weight, as well as the increase of liver weight (Figure 1A,B). After administration of ruscogenin, ruscogenin could obviously increase body weight and decrease liver weight in a dose-dependent manner. The high dosage $20 \mathrm{mg} / \mathrm{kg}$ ruscogenin had the best improvements on the body and liver weight.

\section{Ruscogenin reduced LUC bioluminescent signal in colorectal liver metastasis mice}

LUC was used to track HCT-116-LUC tumor growth over time. LUC imaging identified a higher bioluminescent signal in the tumor inoculation group than that in the control group (Figure 2). From the appearance and size of the tumors, the tumor sizes and volumes were significantly increased in the treatment group, compared with that in control group. The results of pathological section demonstrated that white nodule existed on the surface of liver, which was diagnosed as the metastasis of colon cancer. Not only the average liver quality was different, but also the tumor nodule was different with significant statistic difference. The cancer cells on the liver grew into masses with white nodules. At higher magnification, cancer tumor region had obvious nuclear pyknosis, partial nuclear disintegration, and cellular apoptosis. Notably, the administration of ruscogenin could reduce fluorescence intensity within the tumor region in a dose-dependent manner (Figure 3). The biggest tumor nodules dropped on the liver could be seen after the administration of high dosage $20 \mathrm{mg} / \mathrm{kg}$ ruscogenin group. The red LUC imaging region was decreased after the intervention of ruscogenin 

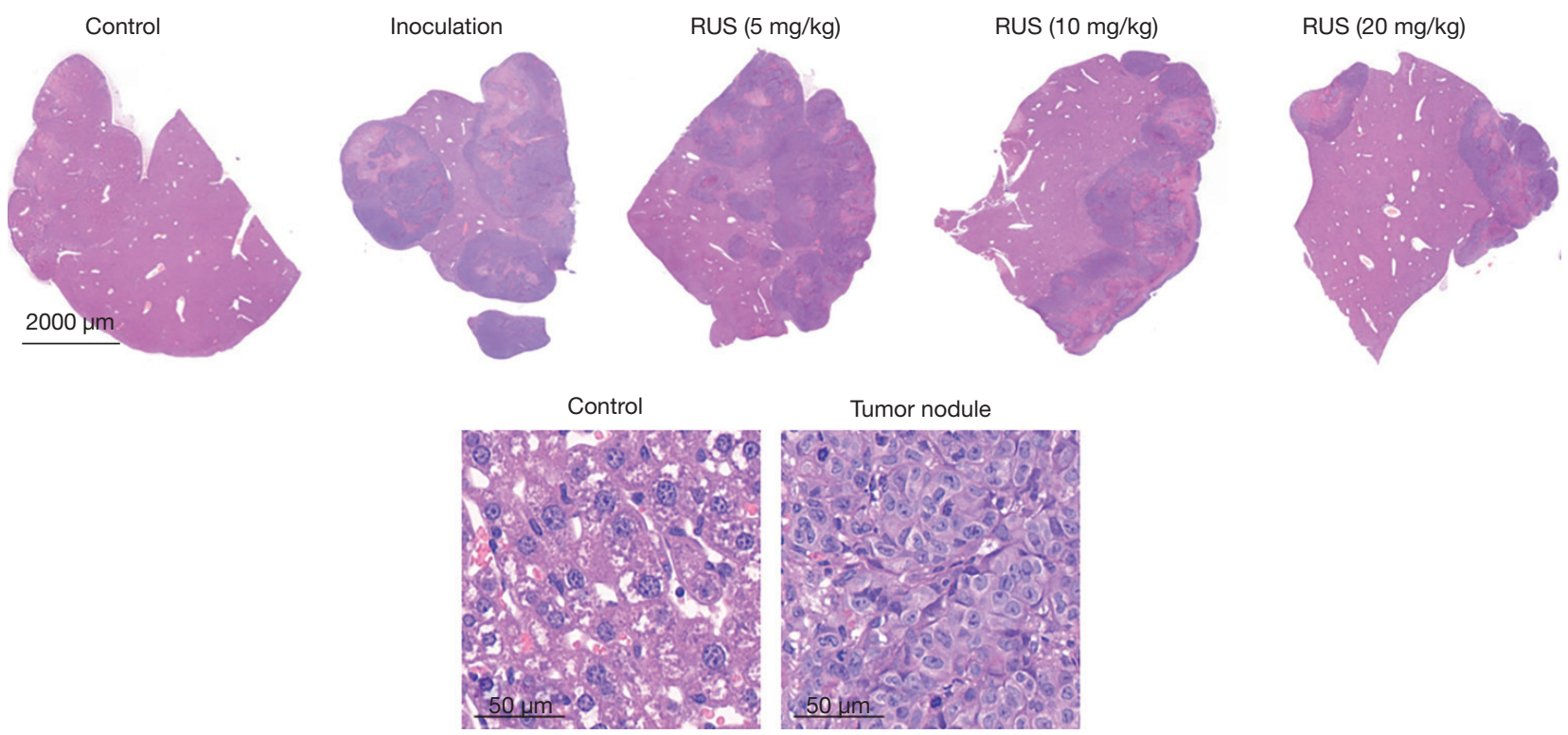

Figure 3 Ruscogenin reduced the acreage of white tumor nodule on liver. Bar: upper =2,000 $\mu \mathrm{m}$, bottom =50 $\mu \mathrm{m}$.

(Figure 2), while the high dose $20 \mathrm{mg} / \mathrm{kg}$ ruscogenin could minimize the growth of tumor. Meanwhile, the administration of ruscogenin could reduce the acreage of white tumor nodule on liver, the high dose ruscogenin could minimize the growth of tumor nodule.

\section{Ruscogenin regulated the expression of tight junction and migration proteins in colorectal liver metastasis mice}

The expression of occludin, claudin-5, and ZO-1 was measured for colorectal liver metastasis changes by western blot. Occludin, claudin-5, and ZO-1 was significantly reduced (claudin-5: $0.67 \pm 0.07, \mathrm{P}<0.01$; occludin: $0.51 \pm 0.05$, $\mathrm{P}<0.01$; ZO-1: $0.58 \pm 0.02, \mathrm{P}<0.01)$ compared with that in the control group (claudin-5: $1.02 \pm 0.06$; occludin: $1.15 \pm 0.06 ; \mathrm{ZO}-1: 1.13 \pm 0.04)$. The protein expression was compared between groups via student's t-test statistical method. TRAP1, ki67, and E-cadherin was abnormally elevated expression compared with that in the control group. TRAP1 was increased $(1.04 \pm 0.03, \mathrm{P}<0.01)$ compared with that in the control group $(0.37 \pm 0.05)$, while E-cadherin was decreased $(0.56 \pm 0.06, \mathrm{P}<0.01)$ compared with that in the control group $(1.17 \pm 0.07)$, and ki67 $(1.19 \pm 0.03, \mathrm{P}<0.01)$ was increased compared with that in the control group $(0.32 \pm 0.04)$. Ruscogenin could rescue the downregulation of tight junction proteins, while upregulated the expression of claudin-5, occludin, and ZO-1 (low dose ruscogenin: claudin-5: 0.70 $\pm 0.03, \mathrm{P}<0.05$; occludin: $0.59 \pm 0.02, \mathrm{P}<0.05$; ZO-1: $0.63 \pm 0.07, \mathrm{P}<0.05$; mediate dose ruscogenin: claudin-5: $0.77 \pm 0.04, \mathrm{P}<0.01$; occludin: $0.63 \pm 0.07, \mathrm{P}<0.01$; ZO-1: $0.70 \pm 0.03, \mathrm{P}<0.01$; high dose ruscogenin: claudin-5: $0.84 \pm 0.04, \mathrm{P}<0.01$; occludin: $0.71 \pm 0.04, \mathrm{P}<0.01$; ZO- 1 : $0.78 \pm 0.04, \mathrm{P}<0.01$ ) (Figure 4). The high dosage ruscogenin had the better attenuation of tight junction proteins than that with the administration of mediate dosage ruscogenin, and the effects of mediate dosage ruscogenin had the better alleviative functions than that in the low dosage ruscogenin group. Ruscogenin could upregulate the expression of E-cadherin (low dose ruscogenin: $0.64 \pm 0.02$, $\mathrm{P}<0.01$; mediate dose ruscogenin: $0.70 \pm 0.03, \mathrm{P}<0.01$; high dose ruscogenin: $0.75 \pm 0.04, \mathrm{P}<0.01)$ and downregulate the expression of ki67 (low dose ruscogenin: $0.88 \pm 0.04$, $\mathrm{P}<0.05$; mediate dose ruscogenin: $0.76 \pm 0.06, \mathrm{P}<0.01$; high dose ruscogenin: $0.57 \pm 0.03, \mathrm{P}<0.01$ ) and TRAP1 (low dose ruscogenin: $0.94 \pm 0.02, \mathrm{P}<0.05$; mediate dose ruscogenin: $0.85 \pm 0.04, \mathrm{P}<0.01$; high dose ruscogenin: $0.71 \pm 0.06$, $\mathrm{P}<0.01$ ) (Figure 4).

\section{Ruscogenin attenuated organ indicators and tumor growth via regulating the expression of TRAP1 in colorectal liver metastasis mice}

The administration of TRAP1 into the colorectal liver metastasis mice could affect organ indicators and tumor 


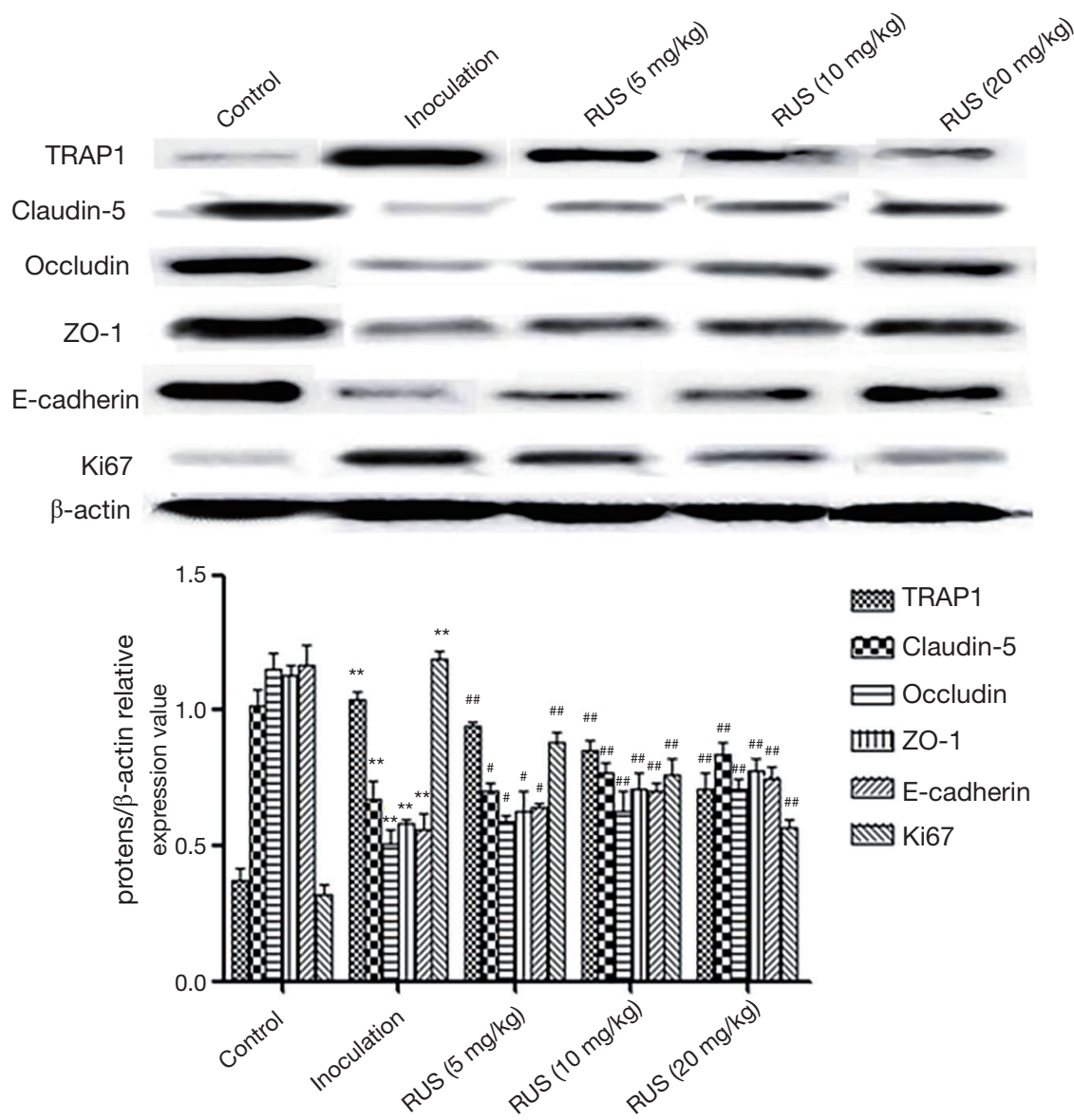

Figure 4 Ruscogenin regulated the expression of tight junction and migration proteins in colorectal liver metastasis mice. The expression change of TRAP1, occludin, claudin-5, ZO-1, E-cadherin, and ki67 of liver in colorectal liver metastasis model. Gel images were cropped to retain only the sample images. The results were expressed as five independent experiments. The data were averages with $\mathrm{SD}$, ${ }^{*} \mathrm{P}<0.05$, ${ }^{* *} \mathrm{P}<0.01$ vs. control group, ${ }^{\#} \mathrm{P}<0.05,{ }^{\# \#} \mathrm{P}<0.01$ vs. inoculation group. TRAP1, tumor necrosis factor receptor associated protein $1 ; \mathrm{ZO}-1$, zonula occludens 1 .

growth. In consideration of high dosage ruscogenin on the attenuation of colorectal liver metastasis, we used high dosage $20 \mathrm{mg} / \mathrm{kg}$ ruscogenin for further research. Downregulation of TRAP1 could increase body weight and decrease liver weight, while ruscogenin had little effects on the body weight and liver weight already defused by the administration of TRAP1 antagonists (Figure $5 A, B$ ). Also, downregulation of TRAP1 could lower the LUC bioluminescent signal, and reduce the tumor sizes and liver weight, the administration of ruscogenin have been found having the similar effect (Figure 6A,B). Overexpression of TRAP1 could aggravate the deterioration of organs, and increase tumor growth on liver by the administration of TRAP1 agonists.

\section{Ruscogenin attenuated tight junction injury and tumor migration via regulating the expression of TRAP1}

Immunostaining showed the occludin, claudin-5, and ZO-1 was continuous and well organized in the liver cells in the control group. Stimulation with colorectal liver metastasis, occludin, claudin-5, and ZO-1 revealed structural disruptions with re-arrangements compared with that the control group. The fluorescent expression of occludin, claudin-5, and ZO-1was reduced compared with that in the liver cells stimulated by colorectal liver metastasis (Figure 7). The administration of overexpression of TRAP1 into the colorectal liver metastasis mice could affect the tight junction injury and tumor migration, while the administration of $20 \mathrm{mg} / \mathrm{kg}$ ruscogenin could alleviate 

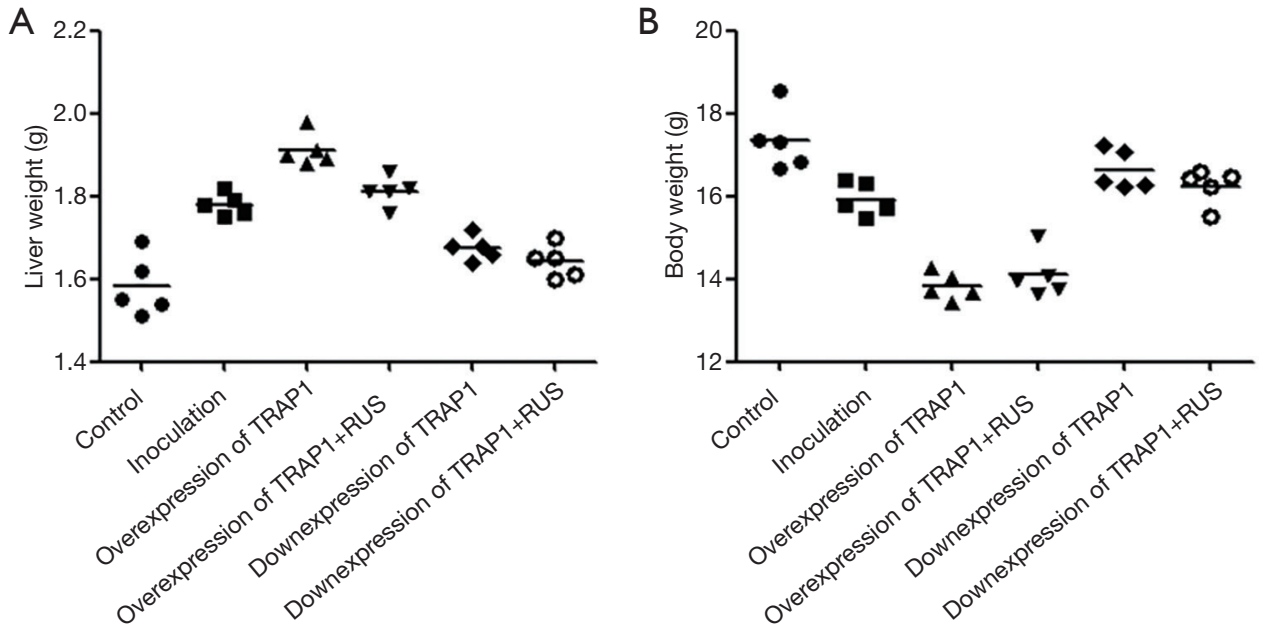

Figure 5 Ruscogenin attenuated body and liver weight in colorectal liver metastasis mice. (A) Ruscogenin attenuated body weight in colorectal liver metastasis mice. (B) Ruscogenin attenuated liver weight in colorectal liver metastasis mice.

A

Control

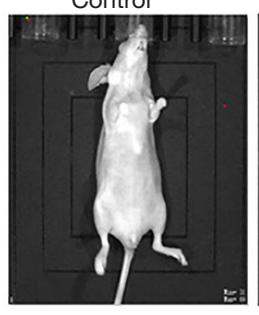

B

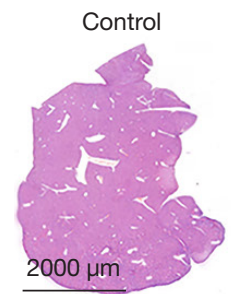

Inoculation

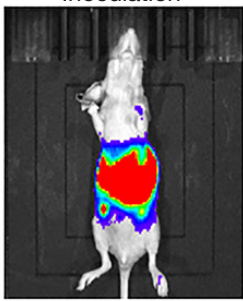

Inoculation

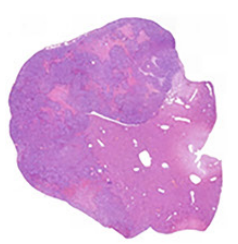

Overexpression of TRAP1

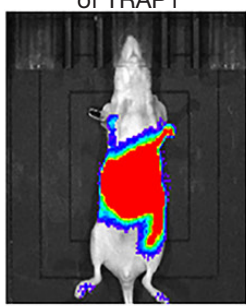

Overexpression of TRAP1

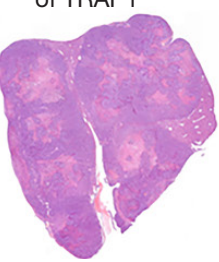

Overexpression of TRAP1+RUS (20 mg/kg)
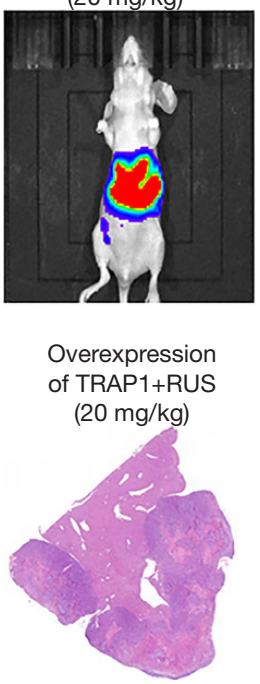

Downexpression of TRAP1

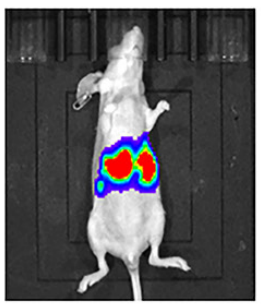

Downexpression of TRAP1

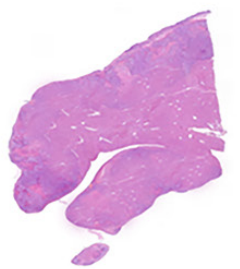

Downexpression of TRAP1+RUS

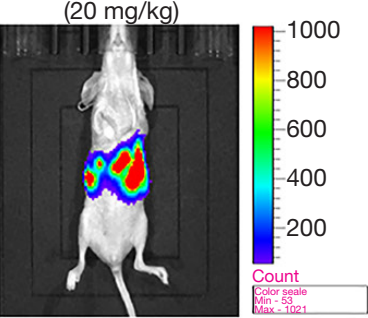

Downexpression of TRAP1+RUS (20 mg/kg)

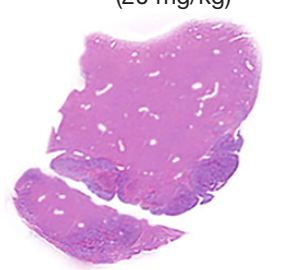

Figure 6 Ruscogenin attenuated tumor growth of liver in colorectal liver metastasis mice. (A) Ruscogenin reduced luciferase bioluminescent signal in colorectal liver metastasis mice. (B) Ruscogenin suppressed tumor growth of liver in colorectal liver metastasis mice. Ruscogenin reduced the acreage of white tumor nodule on liver. $\mathrm{Bar}=2,000 \mu \mathrm{m}$.

the phenomenon caused by the overexpression of TRAP1. The protein expression was compared between groups via student's $t$-test statistical method. Downexpression of TRAP 1 could alleviate the tight junction injury (claudin-5: $0.81 \pm 0.04, \mathrm{P}<0.01$; occludin: $0.74 \pm 0.04$, $\mathrm{P}<0.01$; ZO-1: $0.71 \pm 0.06, \mathrm{P}<0.01$ ), while upexpression of TRAP1 aggravated the tight junction injury (claudin-5:
$0.32 \pm 0.04, \mathrm{P}<0.01$; occludin: $0.36 \pm 0.04, \mathrm{P}<0.01$; ZO-1: $0.36 \pm 0.06, \mathrm{P}<0.01$ ) (Figure 7). The improvement effects of downexpression of TRAP1 could not be further improved after the added of $20 \mathrm{mg} / \mathrm{kg}$ ruscogenin (claudin-5: $0.82 \pm 0.03, \mathrm{P}<0.01$; occludin: $0.79 \pm 0.07, \mathrm{P}<0.01$; ZO-1: $0.73 \pm 0.05, \mathrm{P}<0.01$ ), while the protein expression level had no significant difference (Figure 8). 


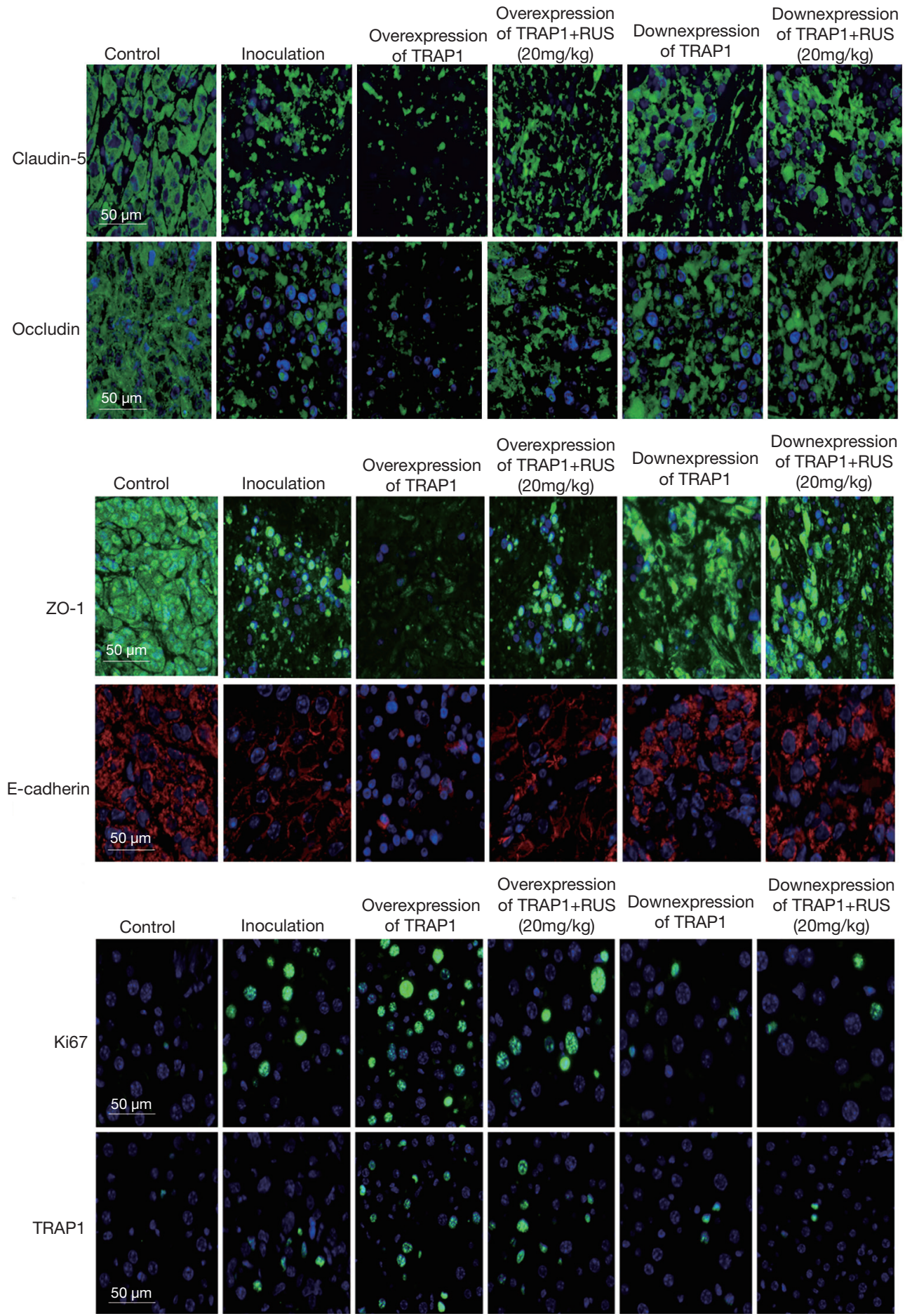

Figure 7 Ruscogenin attenuated the tight junction injury via regulating the expression of TRAP1. The expression of TRAP1, occludin, claudin-5, ZO-1, ki67, and E-cadherin expression in the liver cells stimulated by colorectal liver metastasis via regulating the expression of TRAP1. Representative images on TRAP1 (green), occludin (green), claudin-5 (green), ZO-1 (green), E-cadherin (red), and ki67 (green) in the liver tissues stimulated by colorectal liver metastasis were captured by immunofluorescence staining. Bar $=50 \mu \mathrm{m}$. TRAP1, tumor necrosis factor receptor associated protein 1; ZO-1, zonula occludens 1 . 


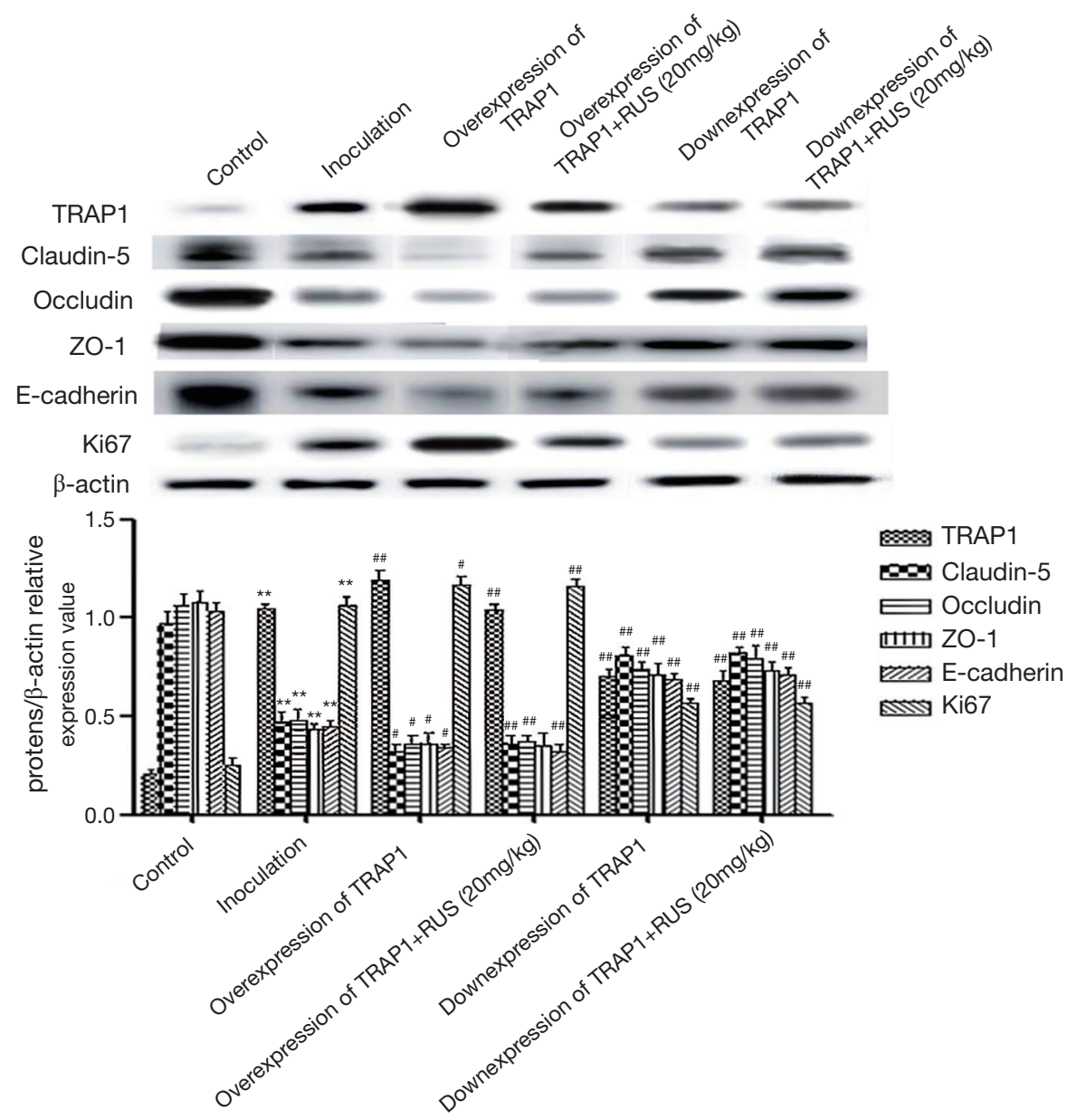

Figure 8 Ruscogenin regulated the expression of tight junction and tumor migration proteins via regulating the expression of TRAP1. The expression of TRAP1, occludin, claudin-5, ZO-1, ki67, and E-cadherin expression in the liver cells stimulated by colorectal liver metastasis via regulating the expression of TRAP1. Gel images were cropped to retain only the sample images. The results were expressed as five independent experiments. The data were averages with $\mathrm{SD},{ }^{*} \mathrm{P}<0.05,{ }^{* *} \mathrm{P}<0.01$ vs. control group, ${ }^{\#} \mathrm{P}<0.05,{ }^{\# \#} \mathrm{P}<0.01$ vs. inoculation group. TRAP1, tumor necrosis factor receptor associated protein 1 ; ZO-1, zonula occludens 1 .

ki67 could predict the increased risk of cancer metastasis formation, while E-cadherin engagement stimulated proliferation. The E-cadherin demonstrated the high brightness immunocytochemical staining proteins along the liver cells border, while E-cadherin showed the little immunocytochemical staining in the control group. ki67 had abnormally elevated expression in colorectal liver metastasis compared with that in the control group (Figure 7). The administration of TRAP1 agonists could enhance tumor migration onto liver (E-cadherin: $0.34 \pm 0.02$, $\mathrm{P}<0.01$; ki67: $1.17 \pm 0.04, \mathrm{P}<0.01$ ), while the administration of TRAP1 antagonists suppressed the tumor migration onto liver (E-cadherin: $0.69 \pm 0.03, \mathrm{P}<0.01$; ki67: 0.57 \pm 0.02 ,
$\mathrm{P}<0.01)$. Ruscogenin could inhibit the aggravate tumor migration by the administration of TRAP1 agonists, while downregulated the expression of ki67 and upregulated the expression of E-cadherin (E-cadherin: $0.32 \pm 0.04, \mathrm{P}<0.01$; ki67: $1.16 \pm 0.04, \mathrm{P}<0.01)$. Ruscogenin had the approximate similar functions as the TRAP1 antagonists on the improvement of tumor migration (E-cadherin: 0.71 \pm 0.04 , $\mathrm{P}<0.01$; ki67: 0.57 $\pm 0.03, \mathrm{P}<0.01$ ) (Figure 8).

\section{Discussion}

Liver has a series of complex defense mechanisms, which could not only stabilize the normal liver barrier, but also 
prevent potential pathogenic bacteria or tumor metastasis from adherence. Similarly, when liver suffered from function loss, tumor signal molecules could pass through the adherence barrier to attack the function of liver, causing the risk of complications induced by tumor signal metastasis (16). The distant metastasis organ of colon cancer was mainly liver, about $50 \%$ of patients had liver metastasis before or after operation, including occult liver metastasis, more importantly, only a small part (10-20\%) was suitable for operation and $70 \%$ had recurrence after operation (16). The location of metastasis included colon lymph node, paracolic lymph node, mesenteric vascular lymph node, and the location of mesenteric root lymph node with greater chance. Hematogenous metastasis was usually the invasion of cancer into the capillary venules. Then tumors directly infiltrated surrounding tissues and organs, or fell onto the liver. The liver metastasis nodule demonstrated white or gray white and hard, as observed on H\&E staining (17). Ruscogenin had the antitumor activity on colorectal liver metastasis mice, which could decrease body weight and increase of liver weight in a dose-dependent manner. LUC bioluminescent signal was reduced in the administration of ruscogenin group than that in the inoculation group. Combined with H\&E liver staining, ruscogenin could obviously decrease the acreage of tumor nodule, the high dosage $20 \mathrm{mg} / \mathrm{kg}$ ruscogenin had the strongest functions on the suppression of tumor migration.

Tight junctions (TJs) are important intercellular junction complexes, which are composed of transmembrane proteins, cytoplasmic attachment proteins, and cytoskeleton proteins (18). Transmembrane proteins include occludin, claudin, and jam, while there are three kinds of cytoplasmic attachment proteins, namely, ZO-1, ZO-2, and ZO-3 (8). The lack of structure and function of tight junction protein could interfere with the transmission of signal molecules between cells, facilitating tumor cells immigrating into blood through the gap between cells (19). The expression of claudin- 6 protein and mRNA was related to the differentiation degree and lymph node metastasis of colon cancer tissues, while the expression of claudin-1 protein in colon cancer was significantly lower than that in adenoma and normal control tissues (20). Reduced expression of claudin-7 promoted epithelial-mesenchymal transition in colorectal cancer (4), meanwhile antibody targeting of claudin-4, claudin-1 was considered as the potential prevention treatment for colorectal liver metastasis $(21,22)$. The relative expression levels of the claudin-1, -3 and -4 genes were higher in cancer than that in normal adjacent mucosa (23). Occludin and ZO-1 was investigated on endothelial cells on metastatic colorectal cancer in the liver (24). After the comparison with human hepatocellular carcinomas and human colorectal liver metastases, the scholars got different conclusions, in colorectal metastatic tumors revealed high levels expression of occludin and ZO-1 (25). Therefore, maintaining the structural and functional integrity for liver could prevent tumor metastasis at any stage of the process. The expression of occludin, claudin-5, and ZO-1 was downregulated in liver region of the colorectal liver metastasis model. The downregulation or the loss of three tight junction proteins confirmed the metastasis tumor striking a thorough damage via the breakdown of the liver barrier function in liver. Ruscogenin could restore the tight junction injury, while upregulated the expression of occludin, claudin-5, and ZO-1 in a dose-dependent manner. Little reports provided the referable administration of ruscogenin on the colorectal liver metastasis mice. We referred to the reference (13) of $10 \mathrm{mg} / \mathrm{kg}$ ruscogenin added to the middle cerebral artery occlusion mice. The drug administration range was preliminarily set to 5,10 , and $20 \mathrm{mg} / \mathrm{kg}$. The data showed that all doses ruscogenin had the attenuation effects on the tight junction injury, the morphological the expression of occludin, claudin-5, and ZO-1 became continuous, distinct, and increasing brightness. High dose ruscogenin exerted the better functions than mediate dose, and mediate dose had better function than low dose. Although preliminary research indicated the effective ruscogenin range, the high dose ruscogenin exerted the superior effects on the colorectal liver metastasis mice. The more precise range verification should be further needed to ensure the effectiveness and avoid side effects.

It has been found that TRAP1 was highly expressed in a variety of tumor cells, TRAP1-SIRT3 complex in glioma stem cells increased mitochondrial respiratory capacity and reduced production of reactive oxygen species (26). TRAP1 regulated hypoxia-induced cardiomyocyte apoptosis through a mitochondria-dependent apoptotic pathway mediated by COXII on hypoxic damage in cardiomyocyte (27). The accumulation of TRAP 1 in mitochondria was a key adaptive mechanism, which made cancer cells less sensitive to tumor microenvironment. Cadherin is a kind of adhesion molecule with many subtypes, of which E-cad is a member of its classic subtype. The expression of E-cad and tumor cell infiltration, differentiation, and differentiation could also weaken the intercellular adhesion function, facilitating tumor invasion and metastasis (28). TRAP1 
expression was reduced in tumor metastases and directly correlated with the epithelial marker E-Cadherin, inversely correlating with the transcription factor slug and the matrix metallopeptidases 2 and 9 (29). Knockdown of TRAP1 in a neuronal cell line decreased tyrosine phosphorylation of STAT3, which resulted in a down-regulation of $\mathrm{N}$-cadherin, and affected the adhesive properties of the cells (30). There was not much evidence to explain the correlation of TRAP1 and E-Cadherin. Especially, the function mechanism of TRAP1 has little reported in colorectal liver metastasis.

TRAP1 was upregulated of the liver in colorectal liver metastasis mice compared with that in control group, as well as the upregulation of nuclear proliferation antigen 67 (ki67), while E-Cadherin was downregulated. ki67 was a nuclear antigen related to cell proliferation specificity, which could reflect the activity of cell proliferation (31). The positive rates of ki67 in the focus tissues were higher than those of the adjacent tissues and the control group, and ki67 was highly expressed in the patients with primary liver cancer (32). When previously administration of TRAP1 agonists, overexpression of TRAP1 could aggravate tight junction injury and E-Cadherin damage, while suppression of TRAP1 antagonists could alleviate the tight junction injury and E-Cadherin damage induced by colorectal liver metastasis. Ruscogenin could both suppress the upregulation of TRAP1 induced by colorectal liver metastasis, meanwhile, there were weakly effects in the intervention of ruscogenin on the regulation of TRAP1 after the administration of TRAP1 antagonists. The downexpression of TRAP1 was approximately of the same effects of $20 \mathrm{mg} / \mathrm{kg}$ ruscogenin on colorectal liver metastasis mice. The above results indicated that TRAP1 might locate at the upstream of the claudin-5, occludin, and ZO-1, E-Cadherin, and ki67. Whether the downregulation of TRAP1 or the administration of $20 \mathrm{mg} / \mathrm{kg}$ ruscogenin could relieve the colorectal liver metastasis via the regulation of TRAP1.

It is of great clinical significance for the early prevention and treatment of colorectal cancer metastasis to monitor the changes of a series of tight junctions, predicting the early stage of colorectal cancer metastasis in advance, and then carrying out early antibody or inhibitor intervention. Ruscogenin could relieve the tight junction injury and E-Cadherin damage in colorectal liver metastasis via the regulation of TRAP1. Ruscogenin itself is relatively safe component, which has not caused any side effects in cerebral ischemia mice. However, the safety and effectiveness of three dose of ruscogenin, especially the highest dose really worth to be further research.

\section{Acknowledgments}

Funding: This work was supported by Foundation Project: National Natural Science Foundation of China (No: 81760094, 31860276), China; The Foundation of Jiangxi Provincial Department of Science and Technology Applied research cultivation projects (No. 20181BBG78051, No. 20171BAB215067); The Foundation of Jiangxi Provincial Department of Science and Technology Key Project (No. 20202ACBL206001); Project of Jiangxi health and Family Planning Commission (No: 20203114, 20203095, 20181021), China; Scientific research project of traditional Chinese medicine in Jiangxi Province (No: 2020B0314); Open Project of Key Laboratory of Modern proparation of TCM, Ministry of Education Jiangxi University of Traditional Chinese Medicine (TCM-2019010).

\section{Footnote}

Reporting Checklist: The authors have completed the ARRIVE reporting checklist. Available at http://dx.doi. org/10.21037/tcr-20-2968

Data Sharing Statement: Available at http://dx.doi. org/10.21037/tcr-20-2968

Conflicts of Interest: All authors have completed the ICMJE uniform disclosure form (available at http://dx.doi. org/10.21037/tcr-20-2968). The authors have no conflicts of interest to declare.

Ethical Statement: The authors are accountable for all aspects of the work in ensuring that questions related to the accuracy or integrity of any part of the work are appropriately investigated and resolved. Experiments were performed under a project license (EA19A139) granted by The First Affiliated Hospital of Nanchang University of ethics committee, in compliance with Animal [Scientific Procedures] Act 1986 guidelines for the care and use of animals.

Open Access Statement: This is an Open Access article distributed in accordance with the Creative Commons Attribution-NonCommercial-NoDerivs 4.0 International License (CC BY-NC-ND 4.0), which permits the noncommercial replication and distribution of the article with 
the strict proviso that no changes or edits are made and the original work is properly cited (including links to both the formal publication through the relevant DOI and the license). See: https://creativecommons.org/licenses/by-nc-nd/4.0/.

\section{References}

1. Benson AB, Venook AP, Al-Hawary MM, et al. NCCN guidelines insights: colon cancer, version 2 2018. J Natl Compr Canc Netw 2018;16:359-69.

2. Lin C, Kuo TC, Lin JC, et al. Delivery of polysaccharides from Ophiopogon japonicus (OJPs) using OJPs/chitosan/ whey protein co-assembled nanoparticles to treat defective intestinal epithelial tight junction barrier. Int J Biol Macromol 2020;160:558-70.

3. Li XP, Zheng LZ, Gu JC, et al. Effects of shengmai injection combined with Oxaliplatin in treatment of liver metastasis from colon cancer. J Shanghai Jiaotong Univ 2008;28:552.

4. Wang L. Shengmai injection auxiliary treatment of malignant digestive tract tumor research situation. J Pract Tradit Chin Internal Med 2012;26:72.

5. Zhang Y, Han Y, Zhao Y, et al. DT-13 meliorates TNF$\alpha$-induced vascular endothelial hyperpermeabilityvia non-muscle myosin IIA and the Src/PI3K/Akt signaling pathway. Front Immunol 2017;8:925.

6. Zhai KF, Zheng JR, Tang YM, et al. The saponin D39 blocks dissociation of non-muscular myosin heavy chain IIA from TNF receptor 2, suppressing tissue factor expression and venous thrombosis. Br J Pharmacol 2017;174:2818-31.

7. Chen EY, Mayo SC, Sutton T, et al. Effect of time to surgery of colorectal liver metastases on survival. J Gastrointest Cancer 2021;52:169-76.

8. Gloushankova NA, Rubtsova SN, Zhitnyak IY. Cadherinmediated cell-cell interactions in normal and cancer cells. Tissue Barriers 2017;5:e1356900.

9. Wang K, Li T, Xu C, et al. Claudin-7 downregulation induces metastasis and invasion in colorectal cancer via the promotion of epithelial-mesenchymal transition. Biochem Biophys Res Commun 2019;508:797-804.

10. Agarwal E, Altman BJ, Seo JH, et al.Myc-mediated transcriptional regulation of the mitochondrial chaperone TRAP1 controls primary and metastatic tumor growth.J Biol Chem 2019;294:10407-14.

11. Matassa DS, Agliarulo I, Avolio R, et al. TRAP1 regulation of cancer metabolism: dual role as oncogene or tumor suppressor. Genes (Basel) 2018;9:195.
12. Tang B, Liang $W$, Liao $Y$, et al. PEA15 promotes liver metastasis of colorectal cancer by upregulating the ERK/ MAPK signaling pathway. Oncol Rep 2019;41:43-56.

13. Cao G, Jiang N, Hu Y, et al. Ruscogenin attenuates cerebral ischemia-Induced blood-brain barrier dysfunction by suppressing TXNIP/NLRP3 inflammasome activation and the MAPK pathway. Int J Mol Sci 2016;17:1418.

14. Maddalena F, Sisinni L, Lettini G, et al. Resistance to paclitaxel in breast carcinoma cells requires a quality control of mitochondrial antiapoptotic proteins by TRAP1. Mol Oncol 2013;7:895-906.

15. Chae YC, Angelin A, Lisanti S, et al. Landscape of the mitochondrial hsp90 metabolome in tumours. Nat Commun 2013;4:2139.

16. Jahanafrooz Z, Mosafer J, Akbari M, et al. Colon cancer therapy by focusing on colon cancer stem cells and their tumor microenvironment. J Cell Physiol 2020;235:4153-66.

17. Teng S, Li YE, Yang M, et al. Tissue-specific transcription reprogramming promotes liver metastasis of colorectal cancer. Cell Res 2020;30:34-49.

18. Lv Y, Liu W, Ruan Z, et al. Myosin IIA regulated tight junction in oxygen glucose deprivated brain endothelial cells via activation of TLR4/PI3K/Akt/JNK1/2/14-3-3e/ $\mathrm{NF}-\kappa \mathrm{B} / \mathrm{MMP} 9$ signal transduction pathway. Cell Mol Neurobiol 2019;39:301-19.

19. Bhat AA, Uppada S, Achkar IW, et al. Tight junction proteins and signaling pathways in cancer and inflammation: a functional crosstalk. Front Physiol 2019;9:1942.

20. Kim H, Kim SH, Hwang D, et al. Extracellular pyruvate kinase M2 facilitates cell migration by upregulating claudin-1 expression in colon cancer cells. Biochem Cell Biol 2020;98:219-26.

21. Fujiwara-Tani R, Sasaki T, Luo Y, et al. Anti-claudin-4 extracellular domain antibody enhances the antitumoral effects of chemotherapeutic and antibody drugs in colorectal cancer. Oncotarget 2018;9:37367-78.

22. Cherradi S, Ayrolles-Torro A, Vezzo-Vié N, et al. Antibody targeting of claudin-1 as a potential colorectal cancer therapy. J Exp Clin Cancer Res 2017;36:89.

23. Oshima T, Kunisaki C, Yoshihara K, et al. Reduced expression of the claudin-7 gene correlates with venous invasion and liver metastasis in colorectal cancer. Oncol Rep 2008;19:953-59.

24. Ueno Y, Ozaki S, Umakoshi A, et al. Chloride intracellular channel protein 2 in cancer and non-cancer human tissues: relationship with tight junctions. Tissue Barriers 2019;7:1593775. 
25. Orbán E, Szabó E, Lotz G, et al. Different expression of occludin and ZO-1 in primary and metastatic liver tumors. Pathol Oncol Res 2008;14:299-306.

26. Park HK, Hong JH, Oh YT, et al. Interplay between TRAP1 and sirtuin-3 modulates mitochondrial respiration and oxidative stress to maintain stemness of glioma stem cells. Cancer Res 2019;79:1369-82.

27. Xiang F, Ma SY, Lv YL, et al. Tumor necrosis factor receptor-associated protein 1 regulates hypoxia-induced apoptosis through a mitochondria-dependent pathway mediated by cytochrome c oxidase subunit II. Burns Trauma 2019;7:16.

28. Druzhkova I, Ignatova N, Prodanets N, et al. E-Cadherin in Colorectal Cancer: Relation to Chemosensitivity. Clin Colorectal Cancer 2019;18:e74-86.

Cite this article as: $\mathrm{Lv} \mathrm{Y}, \mathrm{Wu} \mathrm{X}$, Chen J, Shao F. Ruscogenin attenuated tight junction injury and tumor migration in colorectal liver metastasis mice via regulating TRAP1. Transl Cancer Res 2021;10(3):1470-1483. doi: 10.21037/tcr-20-2968
29. Amoroso MR, Matassa DS, Agliarulo I, et al. TRAP1 downregulation in human ovarian cancer enhances invasion and epithelial-mesenchymal transition. Cell Death Dis 2016;7:e2522.

30. Kubota K, Inoue K, Hashimoto R, et al. Tumor necrosis factor receptor-associated protein 1 regulates cell adhesion and synaptic morphology via modulation of N-cadherin expression. J Neurochem 2009;110:496-508.

31. Xiao Y, Zhang Y, Xiao F. Comparison of several commonly used detection indicators of cell senescence. Drug Chem Toxicol 2020;43:213-8.

32. Xu LX, He MH, Dai ZH, et al. Genomic and transcriptional heterogeneity of multifocal hepatocellular carcinoma. Ann Oncol 2019;30:990-7. 